\title{
ESTIMATIVA DE ÁREAS AGRÍCOLAS MUNICIPAIS, UTILIZANDO SISTEMA DE AMOSTRAGEM SIMPLES SOBRE IMAGENS DE SATÉLITE(1)
}

\author{
JOSÉ CARLOS NEVES EPIPHANIO(2); ALFREDO JOSÉ BARRETO LUIZ(3); \\ ANTONIO ROBERTO FORMAGGIO ${ }^{(2)}$
}

\begin{abstract}
RESUMO
Estimativas da área cultivada são importantes para diversas aplicações. Entretanto, como os campos de cultivo são distribuídos irregularmente, os levantamentos agrícolas tornam-se operações complexas e dispendiosas. As imagens de satélite, por possuírem caráter de cobertura abrangente e serem obtidas repetidamente, podem auxiliar no levantamento da área ocupada pelas culturas, municipal ou nacional. Propõe-se um método amostral aleatório simples para a quantificação de áreas de culturas expressivas no município de Ipuã, SP. Com base na interpretação não exaustiva das áreas de soja e milho, de uma imagem Landsat-5, de janeiro de 2000, contabilizaram-se as áreas parciais de soja e de milho como $26,5 \%$ e $3 \%$ do município respectivamente. Amostraram-se 521 pontos sobre a imagem, aos quais foi atribuída a classe de uso: soja, milho, "não-soja ou milho". Pela simulação de subconjuntos variando de 10 a 500 pontos, e assumindo uma distribuição hipergeométrica, pôde-se avaliar o nível de variabilidade das estimativas em função dos tamanhos de amostras. Para a soja, observou-se que a média de um conjunto de 10 amostras de 250 pontos amostrais superestimava a área medida em 3\%. Mostra-se, também, que quando a área cultivada com soja é 35\% do município, para um nível de significância de $90 \%$ e com 500 pontos amostrais a porcentagem de área estimada é de 31,4\% e 38,4\% do município. A amostragem, com base na estrutura das imagens de satélite, permite um levantamento ágil e com conhecida precisão da área cultivada com grandes culturas no município.

Palavras-chave: Landsat, sensoriamento remoto, agricultura, estatística agrícola, safra, soja, milho.
\end{abstract}

\section{ABSTRACT \\ CROP AREA ESTIMATES USING SIMPLE SAMPLING SCHEME ON SATELLITE IMAGES}

Crop area estimates are important for several applications. However, as the crop fields are irregularly distributed, such surveys become a difficult and expensive operation. Remote sensing satellite images, for having a broad coverage and being obtained continuously, can be very helpful. In this paper a simple sampling method for quantifying the main crops in a municipality level is described. A non-exhaustive interpretation of soybean, corn and "non-corn or non-soybean" was performed on a TM/Landsat-5 image, and a random sampling of 521 pixels was selected. The areas of soybean and corn were $26.5 \%$ and $3.0 \%$ of the municipality, respectively. By simulating sets varying from 10 to 500 sampling points each, and assuming a hypergeometric distribution, it was possible to evaluate the variability of the estimation as a function of the sampling size. It was observed that for a sampling size of 250 pixels there was an overestimation of $3 \%$ for soybean and an underestimation of $1 \%$ for corn. It is also shown that when a crop occupies $35 \%$ of the municipality, for a significance level of $90 \%$, and for 500 sampling pixels, the estimation results ranges between $31.4 \%$ and $38.4 \%$ of the municipality area. The sampling based on the satellite image structure allows a fast and fairly precise survey of the more important cultivated crop area of a municipality. Key words: remote sensing, agricultural survey, soybean, corn, Landsat, statistics.

$\left({ }^{1}\right)$ Trabalho parcialmente financiado pela FAPESP. Recebido em 28 de dezembro de 2001 e aceito em 5 de agosto de 2002.

$\left(^{2}\right)$ Instituto Nacional de Pesquisas Espaciais (INPE), Divisão de Sensoriamento Remoto, Av. dos Astronautas, 1.758, 12227-010 São José dos Campos (SP). E-mail: epiphani@ltid.inpe.br, formag@ltid.inpe.br

(3) Instituto Nacional de Pesquisas Espaciais (INPE). Pesquisador da EMBRAPA/CNPMA. E-mail: barreto@ltid.inpe.br 


\section{INTRODUÇÃO}

A estimativa da área das culturas agrícolas é uma importante variável no estabelecimento de instrumentos de políticas e negócios agrícolas. Muitas decisões sobre importações ou exportações, manutenção de estoques reguladores, estratégias para os diversos insumos, alocação de créditos e outros aspectos são estabelecidas com base nas estatísticas agrícolas (PINO, 1999). A obtenção de informação atualizada sobre esse setor da economia é particularmente difícil por causa das características inerentes às atividades agropecuárias, como a ampla dispersão espacial, o grande número de produtores, a variabilidade do tamanho das propriedades e talhões, a inexistência ou fragilidade da associação entre produtores, etc. Segundo IPPOLITI-RAMiLo et al. (1999), o caráter multi-espectral, sinóptico, repetitivo e global dos dados orbitais indica essa tecnologia como potencial aliada dos sistemas de estimativas agrícolas. Segundo a FAO (1998), já é corriqueiro o uso de imagens de sensoriamento remoto como auxiliares nos sistemas nacionais de estimativas agrícolas de diversos países.

Os EUA, por exemplo, com o lançamento ao espaço do primeiro satélite da série Landsat, em 1972, passaram a utilizar os dados de sensoriamento remoto orbital para o aprimoramento dos métodos de amostragem por área e contribuíram para demonstrar o valor das imagens, tanto para a construção dos painéis amostrais, como para o desenvolvimento de um estimador de área cultivada (Allen e Hanuschak, 1988). Uma das primeiras utilidades atribuídas às imagens obtidas por satélites foi a de material suplementar na construção de painéis amostrais por área, particularmente na estratificação de grandes áreas e, dependendo da resolução espacial, também na subdivisão dos estratos nas unidades básicas de amostragem e até mesmo na identificação e delineamento dos seus limites físicos (Wigton e Bormann, 1978). Após terem consolidado uma metodologia para os levantamentos agrícolas domésticos, os Estados Unidos passaram a se preocupar com o aprimoramento das estatísticas agrícolas em âmbito mundial (MACDonAlD e HALL, 1980). Assim, em 1974, iniciaram o experimento chamado LACIE (Large Area Crop Inventory Experiment) com o objetivo de assimilar a tecnologia de sensoriamento remoto, aplicar a metodologia dos levantamentos por amostragem para monitorar a produção de trigo ao redor do mundo e demonstrar a exeqüibilidade técnica e financeira de um sistema demonitoramento agrícola global (Houstone HALL, 1984).
Entre 1980 e 1988, como uma expansão do LACIE, os EUA conduziram o AgRISTARS (Agriculture and Resources Inventory Surveys Through Aerospace Remote Sensing), que favoreceu a incorporação, de forma operacional, do sensoriamento remoto na rotina dos levantamentos agrícolas (Houston e HALL, 1984).

Na Europa, desde 1989, funciona, sob os auspícios da Comunidade Européia (CE), o projeto Monitoramento Agrícola por Sensoriamento Remoto (MARS), cujos objetivos iniciais eram distinguir, identificar e medir a área das culturas agrícolas mais importantes e, em seguida, estimar a produção a tempo de permitir a tomada de decisões (KLERSY, 1992). A principal diferença entre o método usado no MARS e o utilizado nos EUA é que, no primeiro, os contornos das unidades básicas de amostragem são traçados artificialmente na forma de quadrados de $50 \mathrm{~km}$ de lado ou círculos de $25 \mathrm{~km}$ de raio, de maneira a serem compatíveis com uma imagem do satélite SPOT (TERRES et al., 1995), enquanto no segundo eles são definidos por limites físicos no terreno (e utilizam imagens do satélite Landsat). Vossen (1995) citou como resultado do MARS, com respeito à estratificação, o emprego das imagens de satélite como a maneira ideal de detectar áreas homogêneas quanto ao uso da terra, identificar os limites físicos dos segmentos e auxiliar na sua seleção.

Além das ações coordenadas pela $\mathrm{CE}$, diversos países da Europa têm envidado esforços no sentido de utilizar o sensoriamento remoto em programas próprios de levantamento de dados agrícolas. Como exemplo, há o programa AGRIT na Itália (MEYER-Roux e KING, 1992). Outros países também estão incorporando a tecnologia do sensoriamento remoto aos seus levantamentos agrícolas, principalmente devido à pressão da CE por informações padronizadas, como Espanha (FAO, 1998), Grécia (Tsiligirides, 1998), Portugal, Checoslováquia e Romênia (MARS, 1993).

Fora do eixo EUA-Europa existem ainda outros países que utilizam imagens de satélite, principalmente para a construção dos estratos, como é o caso do Marrocos e do Paquistão (FAO, 1998). Já na Índia, há mais de uma década, realizam-se inventários agrícolas utilizando dados de sensoriamento remoto com base em plataformas espaciais. Dados dos satélites indianos de sensoriamento remoto IRS 1A e 1B têm sido usados com sucesso para se obter previsões de produção das culturas de arroz, mostarda e batata (PANIGRAHY AND CHAKRABORTY, 1998); trigo (SRIDHAR et al., 1994) e outras culturas (NAGESWARA RAO and MOHANKUMAR, 1994). Trabalhos que relacionam o sensoriamento remoto $\mathrm{e}$ o monitoramento das atividades agrícolas também 
já foram realizados em regiões tão diferentes como o Egito (Pax-Lenney e Woodcock, 1997), Costa Rica (Mulders et al., 1992), China (Yuliang et al., 2000), Austrália (WALKER e Mallawaarachchi, 1998) e Indonésia (RIBBES AND LETOAN, 1999); neste último, utilizaram-se dados obtidos por radar.

As estatísticas agrícolas oficiais no Brasil são responsabilidade do Instituto Brasileiro de Geografia e Estatística (IBGE) que realiza regularmente o Levantamento Sistemático da Produção Agropecuária (LSPA), método subjetivo e não probabilístico que se baseia em informações fornecidas por agentes locais, geralmente municipais, ligados ao meio rural (IPPOLITI-RAMilo et al., 1999). Visando atingir o que preconiza a FAO (Food and Agriculture Organization of the United Nations), tem havido um esforço por parte de alguns agentes governamentais no sentido de estabelecer um método, com base em modelos amostrais, para os levantamentos agrícolas.

Nessa direção, o IBGE vem aplicando um método amostral para o levantamento agrícola no Estado do Paraná (FAO, 1996; 1998). O método denominado Sistema Amostral de Levantamento Agrícola (PREVS) baseia-se em uma estratificação do Estado segundo o padrão de uso, e na definição de um painel amostral, a partir do qual define-se o número de segmentos a serem visitados em campo. Os produtos de sensoriamento remoto, como imagens de satélite e fotografias aéreas, são utilizados nas fases de estratificação e de levantamento dos segmentos no campo. Em geral, cada segmento é composto de diversos talhões, com múltiplos usos.

A definição de um painel amostral (onde o desenho dos segmentos, com áreas semelhantes, é feito sobre imagens de satélite, e o posterior detalhamento de cada segmento, no campo, por ocasião dos levantamentos propriamente ditos, sobre fotografias aéreas, em geral, defasadas no tempo) é um processo trabalhoso, particularmente em terrenos de topografia mais irregular. Assim, é desejável que se busquem métodos auxiliares ou alternativos para subsidiar o levantamento agrícola.

A agricultura abrange vasta gama de atividades, envolvendo dezenas de espécies vegetais cultivadas. Entretanto, numa região específica, devido às condicionantes ambientais e culturais, apenas algumas espécies são consideradas importantes. Isso é particularmente verdadeiro no âmbito municipal, como constatado, por exemplo, por SANO et al. (1998) em seu trabalho de monitoramento da ocupação agrícola realizado em três municípios no Sul do Estado do Maranhão.
Entre as culturas agrícolas, algumas oferecem maior potencial para serem efetivamente monitoradas pelo sensoriamento remoto. São aquelas que ocupam maior área, cultivadas mecanicamente em talhões com vários hectares. É para a estimativa da área plantada com essas culturas, por município, que se propõe o presente método de amostragem por pontos ou pixels sobre imagens de satélite.

\section{FUNDAMENTAÇÃO TEÓRICA}

Uma imagem de satélite pode ser vista como um universo amostral regular, com os pixels da imagem servindo como elementos amostrais regularmente espaçados, de área fixa, e passíveis de identificação e de localização. O próprio processo de aquisição da imagem produz uma divisão imaginária da superfície terrestre em pixels ou elementos de cena que a recobrem totalmente e sem sobreposição. Nesse caso, o número de pixels pertencente a certa classe determina a proporcionalidade da área ocupada por aquela classe em relação à área da imagem ou do universo amostral.

Assumindo a área de um município qualquer como a região de interesse, existirá um número finito $\mathbf{N}$ de pixels que representará, na imagem, a sua superfície real, conforme a equação 1 :

$$
\mathrm{N}=\frac{\text { Área do município }}{\text { Área do pixel }}
$$

Nesse caso, a população é a área do município e os elementos da amostra correspondem exatamente aos pixels. Como no caso de painéis amostrais por área, a probabilidade de seleção de cada elemento da amostra é proporcional à sua área e, como a área de cada pixel de uma imagem é constante, todo elemento da população tem garantida uma mesma e conhecida chance de pertencer à amostra. Quando corretamente planejado, esse tipo de levantamento assegura que não ocorram falhas de cobertura. Uma das limitações ao uso de esquemas amostrais probabilísticos, por área, com elementos de dimensões arbitrariamente predefinidas, é a necessidade de identificar precisamente seus limites físicos no terreno. Atualmente, a alta precisão, o baixo custo e a facilidade de operação dos aparelhos de GPS (Global Positioning Systems) disponíveis permitem a localização com precisão e rapidez desse elemento amostral. Além disso, um pixel que servirá de elemento amostral agrícola não ocorre isoladamente, mas sim dentro de um talhão constituído de muitos outros pixels, dando ao 
conjunto uma conformação tipicamente poligonal, e isso facilita sua identificação. Outros pontos amostrais, com mesmo padrão e já classificados, também servem de apoio à interpretação de um dado pixel ou talhão.

O método proposto é o da Amostragem Aleatória Simples (AAS), que consiste na seleção de uma amostra de $\mathbf{n}$ elementos de um total populacional de $\mathbf{N}$, de tal forma que qualquer possível amostra de tamanho $\mathbf{n}$ tenha uma mesma probabilidade de ser escolhida. Na prática, enumera-se de 1 até $\mathbf{N}$ os pixels que compõem a imagem da área de interesse e, por um algoritmo de geração de números aleatórios, selecionam-se os $\mathbf{n}$ elementos da amostra.

Uma primeira conseqüência prática da escolha do pixel como unidade básica de amostragem é que se um pixel for escolhido para compor uma amostra, não é necessário localizar exatamente no campo a sua posição. Basta localizá-lo na imagem, identificar o segmento ou talhão que o contém, localizar o segmento no campo (o que é muito mais fácil que localizar apenas um pixel), e atribuir ao pixel a mesma classe verificada nesse segmento.

Definidos o pixel, que tem área conhecida e constante, como elemento amostral, e a classe a que ele pertence, como variável de interesse, segue-se que, a proporção com que ocorre uma classe na amostra passa a equivaler à proporção de área amostral ocupada por essa classe. Por expansão, estima-se a área dessa classe na população (no caso, a área de uma cultura em um município). Em se tratando de uma única cultura $\left(\mathbf{x}_{\mathbf{i}}\right)$, um elemento poderá ser classificado em apenas duas categorias: $\mathbf{E}_{\mathbf{i}}$, se pertencer ao conjunto ocupado pela cultura $\mathbf{x}_{\mathbf{i}}$; $\bar{E}_{i^{\prime}}$ se pertencer ao conjunto não ocupado pela cultura $\mathbf{x}_{\mathbf{i}}$. Para facilitar a exposição do método será assumida a seguinte notação:

$\mathbf{A}=$ número de elementos da classe $\mathbf{E}_{\mathbf{i}}$ na população (a na amostra);

$\mathbf{P}=\mathbf{A} / \mathbf{N}=$ proporção de elementos da classe $\mathbf{E}_{\mathbf{i}}$ na população $(\mathbf{p}=\mathbf{a} / \mathbf{n}$ na amostra);

$\mathbf{Q}=1-\mathbf{P}=$ proporção de elementos da classe $\overline{\mathbf{E}}_{\mathrm{i}}$ na população ( $\mathbf{q}=1$ - $\mathbf{p}$ na amostra);

$\mathbf{f}=\mathbf{n} / \mathbf{N}=$ fraçãoamostral $(1 / \mathbf{f}=\mathbf{N} / \mathbf{n}=$ fator deexpansão).

O processo descrito para a obtenção da amostra é, segundo JoHnson e KoTz (1969), a situação clássica que leva naturalmente a uma distribuição discreta do tipo hipergeométrica. Nesse caso, segundo Cochran (1977), a estimativa amostral de $\mathbf{P}$ é dada diretamente por $\mathbf{p}$; e a estimativa de $\mathbf{A}$ é obtida pela aplicação do fator de expansão, ou seja, $\mathbf{A}=\mathbf{a}(1 / \mathbf{f})$.

Supondo que para cada elemento da população existe uma variável $\mathbf{y}_{\mathbf{i}}$ que assume valor 1 se o elemento pertence à classe $\mathrm{E}_{\mathrm{j}}$, ou valor 0 se o elemento pertence à classe $\overline{\mathbf{E}}_{\mathbf{i}}$, torna-se evidente que o total para a população $(Y)$ é obtido com a equação 2:

$$
\mathrm{Y}=\sum_{\mathrm{i}=1}^{\mathrm{N}} \mathrm{y}_{\mathrm{i}}=\mathrm{A}
$$

em que as médias da população e da amostra podem ser calculadas pelas equações 3 e 4 respectivamente:

$$
\begin{gathered}
\bar{Y}=\frac{\sum_{i=1}^{N} y_{i}}{N}=\frac{A}{N}=P \\
\bar{y}=\frac{\sum_{i=1}^{n} y_{i}}{n}=\frac{a}{n}=p
\end{gathered}
$$

Conseqüentemente, a dificuldade de obter $\mathbf{A}$ e $\mathbf{P}$ pode ser reduzida à simples estimativa do total e da média de uma população cujo $\mathbf{y}_{\mathbf{i}}$ é 1 ou 0 . Sob tais condições pode-se afirmar que a proporção amostral $\mathbf{p}=\mathbf{a} / \mathbf{n}$ é uma estimativa não tendenciosa da proporção populacional $\mathbf{P}=\mathbf{A} / \mathbf{N}$.

Na prática, nos casos em que a fração amostral não excede $5 \%(\mathbf{f}<0,05)$, pode-se considerar que a variância da média amostral, ou seja, a variância de p, tem sua estimativa não tendenciosa dada pela equação 5:

$$
\mathrm{S}_{\mathrm{p}}^{2}=\frac{\mathrm{pq}}{(\mathrm{n}-1)}
$$

O número estimado total de elementos da classe $\mathrm{E}_{\mathrm{i}}$ na população é representado como $\hat{\mathrm{A}}=\mathbf{N p}$, e a estimativa não tendenciosa da sua variância pode ser obtida pela equação 6:

$$
\mathrm{S}_{\mathrm{Np}}^{2}=\frac{\mathrm{N}(\mathrm{N}-\mathrm{n})}{(\mathrm{n}-1)} \mathrm{pq}
$$

Quando se conhece, porém, a verdadeira distribuição de freqüência dos dados, é possível, além da média e da variância, estimar a probabilidade real de ocorrência de cada resultado possível para o processo de amostragem. Com isso, pode-se obter uma boa indicação do tamanho de amostra adequado à identificação de eventos (culturas agrícolas no presente caso) com as mais variadas probabilidades de ocorrência na população. Essa possibilidade será utilizada neste trabalho e descrita mais adiante.

\section{MATERIAL E MÉTODOS}

A área de estudo, com $466,5 \mathrm{~km}^{2}$, localiza-se no Norte do Estado de São Paulo e refere-se ao município de Ipuã (coordenadas: 4800`50” W e 
20²6³0"S), região de abrangência do Escritório de Desenvolvimento Regional (EDR) de Orlândia, e caracterizado por ser eminentemente agrícola. A localização do município no Estado pode ser visualizada na figura 1 , que também apresenta parte de uma cena TM-Landsat, na composição 4-vermelho, 5-verde e 3-azul, representando toda a área de Ipuã em uma época de pleno desenvolvimento agrícola.

Há predomínio de solos férteis (Latossolos Vermelhos), com relevo suave ondulado a plano. $\mathrm{O}$ clima é classificado como $\mathrm{Cw}$. A agricultura é tecnificada, havendo várias áreas com sistemas de irrigação do tipo pivô central. As principais culturas são a soja, o milho e a cana-de-açúcar. É comum a prática de dois ciclos agrícolas por ano, com milho e sorgo após o período normal de safra. Também é possível observar a ocorrência da rotação entre canade-açúcar e uma cultura anual por ocasião da reforma do canavial em alguns talhões.

O presente trabalho concentra-se nas culturas expressivas de verão, particularmente a soja e o milho. Assumindo um universo constituído por certa região, por exemplo, o município, sabe-se com precisão o número de pixels que constitui aquele universo. A partir daí, é possível amostrar um número de pixels a fim de estimar a área de certa classe - a soja, naquele universo.

Utilizou-se uma imagem TM/Landsat-5, órbita/ ponto 220/74, obtida em $8 / 1 / 2000$, que serviu como base para os trabalhos de campo e para a localização e interpretação das amostras selecionadas. Embora o formato da imagem já produzisse razoável nível de georreferenciação, a imagem foi georreferenciada com auxílio de oito pontos de controle obtidos com GPS no campo. Para os processamentos, utilizou-se o software SPRING (INPE, 2000). Para a delimitação do município sobre a imagem, utilizou-se a grade municipal do IBGE, com posterior ajuste dos limites pelas cartas 1:50.000. Com auxílio de trabalhos de campo realizados na região em abril e julho de 2000, realizou-se uma interpretação de talhões típicos de soja e de milho ocorrentes na data da passagem do satélite.

Retirou-se uma única amostra de 521 elementos, que consistiam em pontos com coordenadas geográficas definidas com base em números aleatórios gerados pelo SAS (SAS, 1988). Sobre as imagens, verificou-se a que classe pertencia cada ponto amostral, conforme ilustrado na figura 1; o quadro superior direito e a respectiva legenda mostram esquematicamente talhões que contêm alguns pontos amostrais. No detalhe, ilustram-se alguns pixels, entre os quais um ponto amostral a ser identificado (note-se que o quadro de pixels é ilustrativo e não guarda relação de escala com a imagem). O critério de pertinência foi atribuir o pixel que contivesse o ponto à classe soja ou milho sempre que este pixel possuísse alguma área de sobreposição com um polígono de uma dessas classes, ou seja, os pontos de borda foram definidos como pertencentes às classes de milho ou soja, o que pode ter levado à superestimação da área de culturas. A alternativa (considerar os pontos de borda não pertencentes às classes) levaria a uma subestimação. Essa inconsistência se deve ao fato de que os pixels se apresentam na forma de uma matriz de células quadradas, enquanto os limites dos polígonos, na forma vetorial, não havendo coincidência perfeita entre eles. Uma vez conhecido o número de pontos amostrais em cada classe, foi possível derivar a proporção de cada classe no município. A área derivada pelo método de amostragem foi então comparada com a área interpretada.

A seguir, a partir da amostra total, foram extraídos subconjuntos amostrais aleatórios de 10 a 500 pontos amostrais, variando de 10 em 10, com 10 repetições cada um, a fim de verificar a variabilidade estatística desses esquemas amostrais.

Finalmente, e como complemento da análise, fezse uma avaliação da estimativa da área das culturas tendo por base uma amostra genérica de 50 a 500 pontos, em intervalos de 100 pontos amostrais. Adotaram-se os níveis de significância de 95\% e 90\% para as situações reais das estimativas de cana-deaçúcar (40\% da área do município), de soja (35\%), de milho de verão (21\%), de milho safrinha $(12 \%)$ e de sorgo $(6 \%)$ realizadas pela Secretaria da Agricultura e Abastecimento para a safra 2000/2001. A área total usada foi a do município. Além disso, verificou-se a variabilidade do nível de significância quando se fixava o erro em $\pm 3 \%$ em relação à porcentagem estimada pela Secretaria.

\section{RESULTADOS E DISCUSSÃO}

O procedimento de georreferenciação da imagem com os pontos de controle forneceu um erro de 0,65 pixels, quando se utilizaram oito pontos de controle. A poligonalização das áreas agrícolas forneceu um total de 110 talhões de soja e 12 de milho. As áreas do município ficaram divididas da seguinte forma: área municipal igual a $46.645,4$ ha, sendo $12.338,4$ ha de soja, 1.384, 8 ha de milho e 32.922,2 ha referentes a outros usos. Pela amostragem, dos 521 pontos, 159 foram classificados como soja, 13 como milho e 349 como outros usos, correspondendo a 14.235,4; 1.163,9 e 31.246,2 ha respectivamente. 


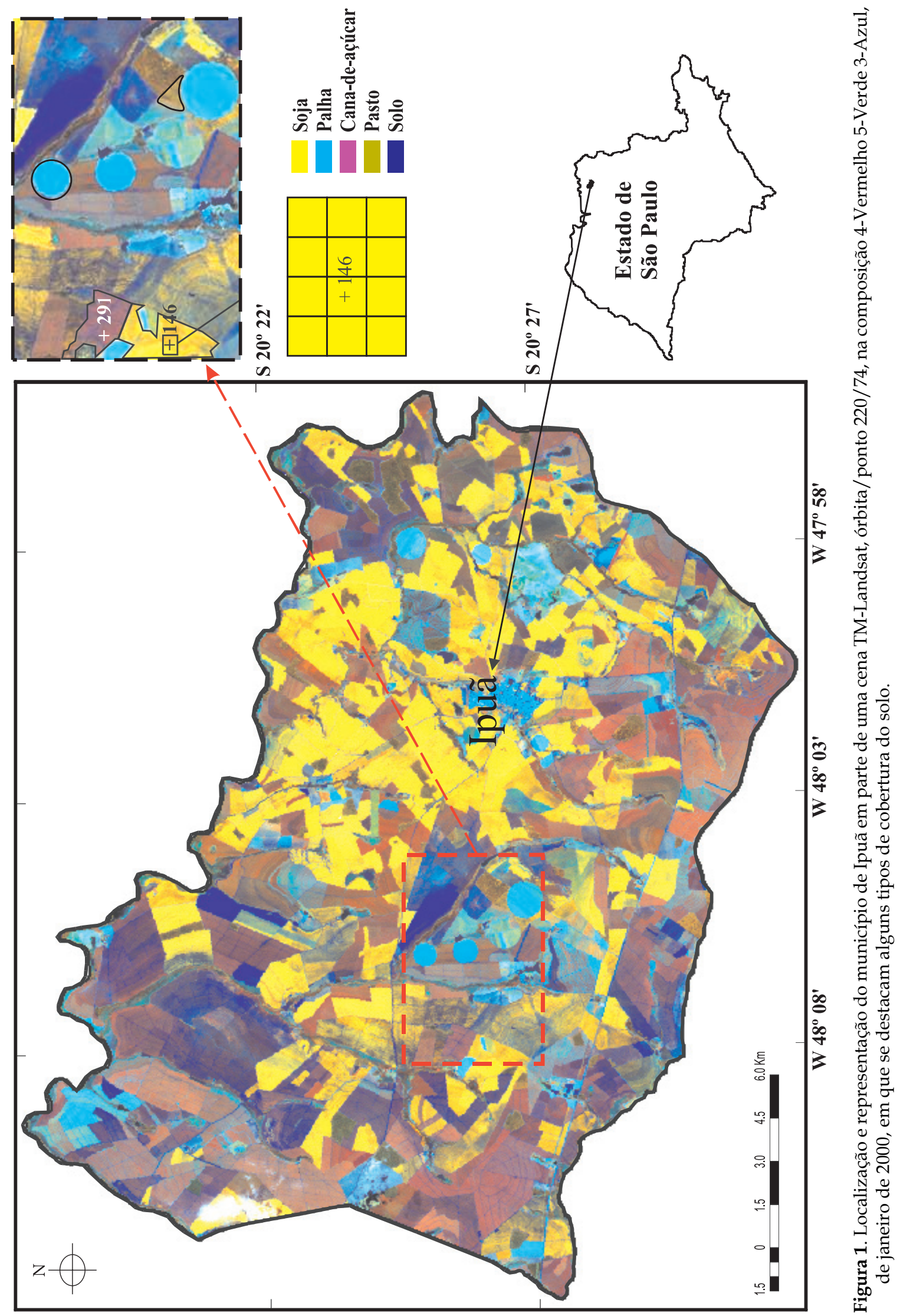


A figura 2 apresenta a diferença entre a porcentagem de área calculada segundo o método amostral e a quantificada pela interpretação (referência). Estão representados as médias (Figura 2a-c) e os coeficientes de variação (Figura 2d-f) de dez amostras de 10 a 500 pontos amostrais, para soja, milho e área que não é nenhuma dessas duas culturas (NMS).

No caso da soja (Figura 2a) observa-se, em amostras de até 50 elementos, que a diferença entre a porcentagem de área calculada e a real (para fins desse trabalho) sofre acentuadas oscilações, até de $8 \%$. Após 50 pontos amostrais há queda nas oscilações e a diferença diminui para valores ao redor de $4 \%$ e mantém-se assim, mesmo com o aumento do número de elementos por amostra. Efeitos semelhantes são observados para milho (Figura $2 b$ ), porém, com valores de diferença oscilando ao redor de $-1 \%$. A área de "não-soja ou não-milho" (NMS, Figura 2c) apresentou o mesmo comportamento, mas com valores de diferença oscilando ao redor de $-3 \%$, pois é, na verdade, o complemento da área de milho e soja. Esses resultados mostram uma superestimativa da área de soja da ordem de 3\%. Nageswara Rao e MOHANKUMAR (1994), também trabalhando sobre imagem do Landsat-TM, estimaram a área ocupada por culturas irrigadas na região de Maddur, distrito de Madya, no Estado de Karnataka na Índia e obtiveram, por amostragem, valores $6,95 \%$ superiores aos encontrados pela enumeração total das áreas irrigadas na região de estudo (27.697 ha contra 25.896 ha respectivamente).

Quanto aos coeficientes de variação para 10 amostras (Figura 2), observa-se que à medida que aumenta o número de pontos amostrais, diminui a variabilidade interamostras. Essa queda do coeficiente de variação $(\mathrm{CV})$ é muito acentuada em amostras até de 50 elementos. A partir daí, embora o coeficiente de variação continue em queda com o aumento dos pontos amostrais, a taxa de queda diminui. Porém, o coeficiente de variação, para um mesmo número de
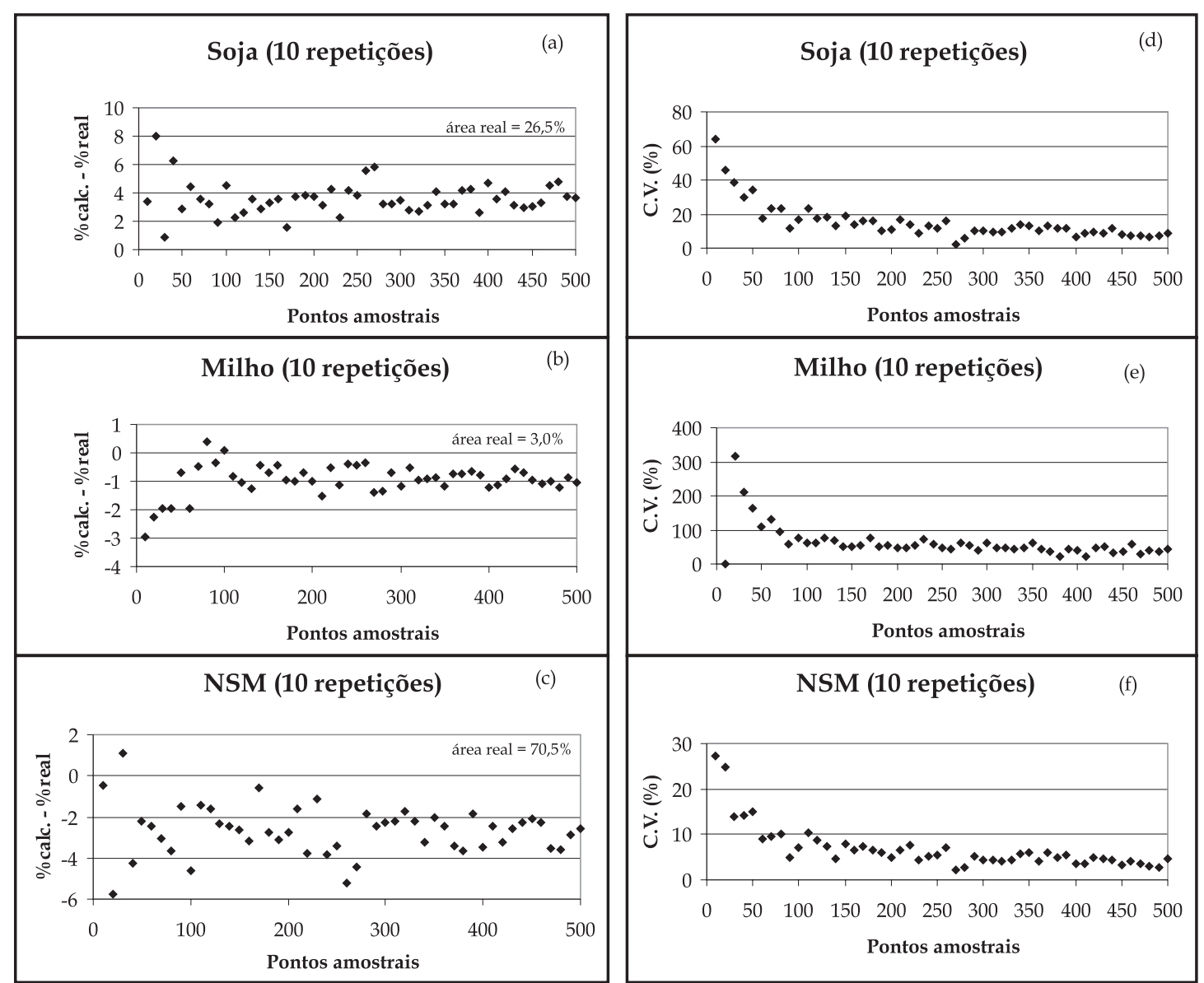

Figura 2. Média de dez amostras para diferença entre as porcentagens de área calculada e quantificada para soja (a), milho (b) e não-milho ou não-soja (NMS) (c); e coeficientes de variação para dez amostras com número variável de elementos amostrais para soja (d), milho (e) e não-milho ou não-soja (NMS) (f). 
elementos na amostra, varia em função do percentual de área ocupada pelas culturas. Para a soja, aproximadamente $26,5 \%$ da área municipal, quando as amostras são de 200 elementos, o coeficiente de variação de dez amostras é cerca de 10\% (Figura 2d), enquanto para o milho (3,0\% da área municipal) o CV é de 50\% (Figura 2e) e, para a área de não-milho ou não-soja (70,6\% do município), de 5\% (Figura 2f). Para efeito de comparação, MACDONALD e HALL (1980) afirmam que, no projeto LACIE, as estimativas de área plantada com trigo na região das Grandes Planícies Norte-Americanas apresentaram coeficientes de variação de 5,7\% em 1975, 4,0\% em 1976 e 2,4\% em 1977.

Outra abordagem de análise necessária e complementar, feita neste trabalho, é a que se consideram os dados da amostra ou mesmo informações a priori para o cálculo do intervalo de confiança da estimativa da população. Nesse caso, os resultados para a análise dos intervalos de confiança ao utilizar as estimativas de áreas dos diversos usos estão no quadro 1. Assim, para a cultura do milho de verão, que, segundo informações do escritório regional de
Orlândia, da Secretaria da Agricultura e Abastecimento do Estado de São Paulo, para a safra 2000/ 2001 , ocupa $21 \%$ da área do município, se forem tomadas amostras com 300 elementos amostrais é esperado que em $95 \%$ das vezes atinja resultados indicativos de que a área esteja entre 16,3\% e 25,5\% (Quadro 1); e que em 90\% dos casos (Quadro 2) tal estimativa esteja entre $17,0 \%$ e $24,8 \%$. Segundo esse procedimento descrito, em $80 \%$ dos casos seria esperada uma estimativa de área entre $21 \% \pm 3 \%$.

O procedimento proposto permite a realização de estimativas na escala municipal, o que é uma evolução em relação ao método da PREVS (FAO, 1996; 1998), que só produz estimativa estadual. Além disso, em alguns métodos que permitem a desagregação da estimativa para áreas menores, há uma sensível perda de precisão. SRIDHAR et al. (1994), por exemplo, registram que para uma região de $5,61 \mathrm{mi}$ lhões de hectares, formada por 7 distritos, no Estado de Madhya Pradesh, na Índia, enquanto a área plantada com trigo, estimada por sensoriamento remoto (1,020 Mha), ficou próxima da área estimada pelo método convencional (1,122 Mha), com CV de 4,9\%,

Quadro 1. Limites inferior (LI) e superior (LS) do intervalo de confiança de 95\% para as estimativas do percentual de área ocupada pelas principais culturas do município de Ipuã (SP), em que se varia o tamanho da amostra

\begin{tabular}{|c|c|c|c|c|c|c|c|c|c|c|}
\hline \multirow{2}{*}{$\begin{array}{c}\text { Tamanho } \\
\text { da amostra }\end{array}$} & \multicolumn{2}{|c|}{ Cana $(40 \%)^{(1)}$} & \multicolumn{2}{|c|}{ Soja (35\%) } & \multicolumn{2}{|c|}{ Milho (21\%) } & \multicolumn{2}{|c|}{ Safrinha $(12 \%)$} & \multicolumn{2}{|c|}{ Sorgo $(6 \%)$} \\
\hline & LI & LS & LI & LS & LI & LS & LI & LS & $\mathrm{LI}$ & LS \\
\hline 50 & 25,6 & 52,8 & 21,0 & 47,5 & 9,2 & 31,8 & 2,6 & 20,8 & 0,0 & 12,4 \\
\hline 100 & 30,0 & 49,2 & 25,3 & 44,0 & 12,8 & 28,8 & 5,4 & 18,2 & 1,2 & 10,6 \\
\hline 200 & 33,0 & 46,6 & 28,2 & 41,4 & 15,2 & 26,5 & 7,4 & 16,4 & 2,6 & 9,3 \\
\hline 300 & 34,3 & 45,4 & 29,5 & 40,3 & 16,3 & 25,5 & 8,3 & 15,6 & 3,3 & 8,6 \\
\hline 400 & 35,1 & 44,7 & 30,5 & 39,6 & 17,0 & 24,9 & 8,8 & 15,2 & 3,6 & 8,3 \\
\hline 500 & 35,6 & 44,2 & 31,0 & 39,1 & 17,4 & 24,5 & 9,1 & 14,8 & 3,9 & 8,1 \\
\hline
\end{tabular}

$\left({ }^{1}\right)$ Valores entre parênteses equivalem à estimativa da Secretaria da Agricultura e Abastecimento do Estado de São Paulo para a safra 2000/2001.

Quadro 2. Limites inferior (LI) e superior (LS) do intervalo de confiança de $90 \%$ para as estimativas do percentual de área ocupada pelas principais culturas do município de Ipuã (SP), em que se varia o tamanho da amostra.

\begin{tabular}{ccccccccccc}
\hline \multirow{2}{*}{$\begin{array}{c}\text { Tamanho } \\
\text { da amostra }\end{array}$} & \multicolumn{2}{c}{ Cana $(40 \%)^{(1)}$} & \multicolumn{2}{c}{ Soja $(35 \%)$} & \multicolumn{2}{c}{ Milho (21\%) } & \multicolumn{3}{c}{ Safrinha (12\%) } & \multicolumn{2}{c}{ Sorgo (6\%) } \\
\cline { 2 - 10 } & LI & LS & LI & LS & LI & LS & LI & LS & LI & LS \\
\hline 50 & 27,7 & 50,6 & 23,0 & 45,3 & 10,8 & 29,8 & 3,9 & 19,1 & 0,1 & 11,1 \\
100 & 31,5 & 47,6 & 26,7 & 42,5 & 14,0 & 27,4 & 6,3 & 17,0 & 1,8 & 9,7 \\
200 & 34,1 & 45,5 & 29,2 & 40,4 & 16,1 & 25,6 & 8,1 & 15,6 & 3,1 & 8,6 \\
300 & 35,2 & 44,5 & 30,3 & 39,4 & 17,0 & 24,8 & 8,8 & 15,0 & 3,7 & 8,2 \\
400 & 35,9 & 43,9 & 31,0 & 38,8 & 17,6 & 24,3 & 9,3 & 14,6 & 4,0 & 7,9 \\
500 & 36,3 & 43,5 & 31,4 & 38,4 & 17,9 & 23,9 & 9,6 & 14,3 & 4,2 & 7,7 \\
\hline
\end{tabular}

$\left({ }^{1}\right)$ Valores entre parênteses equivalem à estimativa da Secretaria da Agricultura e Abastecimento do Estado de São Paulo para a safra 2000/2001. 
Quadro 3. Probabilidade de observação de valores entre mais ou menos 3\% das estimativas do percentual da área ocupada pelas principais culturas do município de Ipuã (SP), em que se varia o tamanho da amostra.

\begin{tabular}{cccccc}
\hline \multirow{2}{*}{$\begin{array}{c}\text { Tamanho } \\
\text { da amostra }\end{array}$} & ${\text { Cana }(40 \%)^{(1)}}^{(37 \text { a } 43 \%)}$ & Soja (35\%) & Milho (21\%) & Safrinha (12\%) & Sorgo (6\%) \\
\hline 50 & 32,7 & 32 a 38\%) & $(18$ a 24\%) & $(9$ a 15\%) & $(3$ a 9\%) \\
100 & 45,7 & 43,8 & 38,6 & 45,5 & 56,8 \\
200 & 61,2 & 62,4 & 53,2 & 73,3 & 78,0 \\
300 & 71,0 & 72,3 & 79,7 & 80,5 & 92,6 \\
400 & 77,9 & 79,1 & 85,9 & 88,9 & 97,2 \\
500 & 82,9 & 84,0 & 90,0 & 93,5 & 98,9 \\
\hline
\end{tabular}

(1) Os valores entre parênteses equivalem à estimativa da Secretaria da Agricultura e Abastecimento do Estado de São Paulo para a safra 2000/2001.

o enfoque amostral adotado não produziu estimativas confiáveis no nível distrital, com seus elevados erros amostrais refletindo-se em altos valores dos coeficientes de variação, entre $9,5 \%$ e $17,7 \%$.

Nesse contexto, o quadro 3 apresenta os níveis de significância que seriam atingidos para as diversas proporções de ocupação da área municipal pelas culturas e para as diversas densidades de amostragem, quando se estabelece o limite de 3\% acima ou abaixo da porcentagem de área ocupada pelas culturas. Por exemplo, com amostras constituídas de 500 elementos amostrais é possível afirmar que em $82,9 \%$ dos casos será feita uma estimativa de cana-de-açúcar entre $40 \% \pm 3 \%$ da área municipal.

Além de adequado às estimativas no âmbito municipal, o método aqui exposto pode ser expandido para grandes áreas oferecendo ganhos na confiabilidade, exigindo apenas pequeno esforço adicional de interpretação. $\mathrm{O}$ uso de conhecimento de comportamento espectral de alvos agrícolas, aliado a um banco de imagens multitemporais pareadas com dados de campo assegura a interpretação de culturas específicas em um tempo relativamente curto (Ortiz et al., 1997). No trabalho de Nageswara RaO e MOHANKUMAR (1994) afirma-se que para a realização de um levantamento de campo, que resultou na identificação de 22.520 ha cultivados com trigo, foram gastos mais de 10 dias, enquanto para a enumeração completa das áreas correspondentes em uma imagem, consumiram-se de 11 a 12 horas, e na amostragem sobre a imagem, empregaram-se apenas duas a três horas.

Outra vantagem é que eventuais pontos de dúvida na classificação podem ser facilmente sanados com visita a campo auxiliada por GPS. Deve-se ressaltar que o uso de GPS para atribuição do ponto amostral a uma classe é muito facilitado em vista de que tal ponto amostral pertence a um talhão muito maior que a unidade amostral. Isso se baseia em um dos pressupostos básicos do sensoriamento remoto agrícola, ou seja, o de que um pixel agrícola é cercado por muitos pixels da mesma natureza (Luz et al., 2002).

Esse método, em que se cria um painel amostral de pixels que estão inseridos em limites compostos de classes de uso, permite rápida avaliação do volume de área de uma certa classe de uso. É importante ressaltar que sua aplicação é mais dirigida a classes agrícolas que ocupem parte significativa do total do município ou região. Além disso, mesmo que numa determinada fase de interesse de levantamento não se disponha de imagens, ainda sim, um levantamento de campo expedito permite o levantamento.

\section{CONCLUSÕES}

1. O método apresentado para a estimativa da área agrícola de soja e milho, a partir de uma simulação, tomando situações reais de distribuição e formato de talhões, mostrou-se muito eficiente. Os desvios das estimativas de área foram sistemáticos e opostos entre as culturas da soja (superestimativa) e do milho (subestimativa).

2. Embora as estimativas de área tenham melhorado com o aumento da densidade amostral, houve uma indicação de que com uma amostra de cerca de 300 elementos amostrais atinge-se razoável nível de estimativa, demonstrado por uma tendência de estabilização do coeficiente de variação.

3. O método de uso do sistema amostral sobre imagens aqui descrito pode ser melhorado em muitos aspectos, notadamente no que diz respeito à interpretação prévia de modo a reduzir o universo amostral.

4. Visto que há uma razoável manutenção do padrão de uso ao longo dos anos, é possível ajustar a amostragem a níveis de significância predefinidos.

5. A precisão do método varia com a expressão territorial da cultura na área de estudo. 


\section{AGRADECIMENTOS}

À Fundação de Amparo à Pesquisa do Estado de São Paulo (FAPESP), pelo financiamento do projeto de número 99/09485-8, do qual se originou este trabalho, e aos revisores anônimos, pelas sugestões que efetivamente contribuíram para maior clareza e correção do texto.

\section{REFERÊNCIAS BIBLIOGRÁFICAS}

ALLEN, J.D.; HANUSCHAK, G.A. The remote sensing applications program of the National Agricultural Statistical Service: 1980-1987. Washington: USDA/ NASS, Research and Applications Division, 1988. 43p. (SRB Staff Report n. ${ }^{\circ}$ SRB-88-08)

COCHRAN, W.G. Sampling Techniques. 3.ed. New York: John Wiley \& Sons, 1977. 428p.

FOOD AND AGRICULTURE ORGANIZATION OF THE UNITED NATIONS (FAO). Multiple frame agricultural surveys: agricultural survey programs based on area frame or dual frame (area and list) sample design. Rome: FAO, 1998. v.2, 242p. (FAO Statistical Development Series, 10)

FOOD AND AGRICULTURE ORGANIZATION OF THE UNITED NATIONS (FAO). Multiple frame agricultural surveys: current survey based on area and list sampling methods. Rome: FAO, 1996. v.1, 119p. (FAO Statistical Development Series, 7)

HOUSTON, A.G.; HALL, F.G. Use of satellite data in agricultural surveys. Communications in Statistics: theory and methods, Hamilton, v.13, n.23, p.2857-2880, 1984. (Special Issue on Crop Surveys Using Satellite Data)

INSTITUTO NACIONAL DE PESQUISAS ESPACIAIS (INPE). Software SPRING. São José dos Campos: INPE, 2000. CD-Rom.

IPPOLITI-RAMILO, G.A.; EPIPHANIO, J.C.N.; SHIMABUKURO, Y.E.; FORMAGGIO, A.R. Sensoriamento remoto orbital como meio auxiliar na previsão de safras. Agricultura em São Paulo, São Paulo, v.46, n.1, p.89-101, 1999.

JOHNSON, N.L.; KOTZ, S. Discrete Distributions. New York: John Wiley \& Sons, 1969. 328p.

KLERSY, R. The work and role of the Commission of the European Communities. International Journal of Remote Sensing, London, v.13, n.6-7, p.1035-1058, 1992.

LUIZ, A.J.B.; OLIVEIRA, J.C.; EPIPHANIO, J.C.N.; FORMAGGIO, A.R. Auxílio das imagens de satélite aos levantamentos por amostragem em agricultura. Agricultura em São Paulo, São Paulo, v.49, n.1, p.49$54,2002$.
MAcDONALD, R.B.; HALL, F.G. Global crop forecasting. Science, Washington D.C., v.208, p.670-679, 1980.

MEYER-ROUX, J.; KING, C. Agriculture and forestry. International Journal of Remote Sensing, London, v.13, n.6-7, p.1329-1341, 1992.

MONITORING AGRICULTURE WITH REMOTE SENSING TECHNIQUES (MARS). Crop area estimation of annual crops through area frame sampling based on segments: results obtained in Europe in 1992. Varese: Ispra, 1993. 21p.

MULDERS, M.A.; DE BRUIN, S.; SCHUILING, B.P. Structured approach to land cover mapping of the Atlantic zone of Costa Rica using single date TM data. International Journal of Remote Sensing, London, v.13, n.16, p.3017-3033, 1992.

NAGESWARA RAO, P.P.; MOHANKUMAR, A. Cropland inventory in the command area of Krishnarajasagar project using satellite data. International Journal of Remote Sensing, London, v.15, n.6, p.1295-1305, 1994.

ORTIZ, M.J.; FORMAGGIO, A.R.; EPIPHANIO, J.C.N. Classification of croplands through integration of remote sensing, GIS, and historical database. International Journal of Remote Sensing, London, v.18, n.1, p.95-105, 1997.

PANIGRAHY, S.; CHAKRABORTY, M. An integrated approach for potato crop intensification using temporal remote sensing data. Journal of Photogrammetry and Remote Sensing, Amsterdam, v.53, n.1, p.54-60, 1998.

PAX-LENNEY, M.; WOODCOCK, C.E. Monitoring agricultural lands in Egypt with multitemporal Landsat TM imagery: how many images are needed? Remote Sensing of Environment, New York, v.59, p.522-529, 1997.

PINO, F.A. Estatísticas agrícolas para o século XXI. Agricultura em São Paulo, São Paulo, v.46, n.2, p.71105, 1999.

RIBBES, F.; LE TOAN, T. Rice field mapping and monitoring with RADARSAT .data. International Journal of Remote Sensing, London, v.20, n.4, p.745765, 1999.

SANO, E.E.; ASSAD, E.D.; ORIOLI, A.L. Monitoramento da ocupaçãoagrícola. In: Assad, E.D.;Sano, E.E.(Eds.). Sistema de Informações Geográficas: aplicações na agricultura. 2.ed. Brasília: Embrapa SPI, 1998. p.179-190.

SAS INSTITUTE. SAS language: reference, version 6. Cary, NC, 1988. 1088p.

SRIDHAR, V.N.; DADHWAL, V.K.; CHAUDHARI, K.N.; SHARMA, R.; BAIRAGI, G.D.; SHARMA, A.K. Wheat production forecasting for a predominantly unirrigated region in Madhya Pradesh (India). International Journal of Remote Sensing, London, v.15, n.6, p.1307-1316, 1994. 
TERRES, J.M.; DELINCE, J.; VAN DE STEENE, M.; HAWKINS, A. The use of remote sensing and GIS capabilities to support the reform of the common agricultural policy of the European Community, Remote Sensing Reviews, London, v.12, p.53-60, 1995.

TSILIGIRIDES, T.A. Remote sensing as a tool for agricultural statistics: a case study of area frame sampling methodology in Hellas. Computer and Electronics in Agriculture, Washington D.C., v.20, n.1, p.45-77, 1998.

VOSSEN, P. Early crop production assessment of the European Union: the systems implemented by MARS-STAT project. p.21-51. In: Dallemand, J.F.; Vossen, P. (Eds.) Agrometeorological Models: theory and applications in MARS project. Luxembourg: Ispra, 1995. 246p.

WALKER, P. A.; MALLAWAARACHCHI, T. Disaggregating agricultural statistics using NOAAAVHRR NDVI. Remote Sensing of Environment, New York, v.63, n.1, p.112-125, 1998.

WIGTON, W. H.; BORMANN, P. A guide to area sampling frame construction utilizing satellite imagery. Washington: USDA, Statistical Report Service Staff Report, 1978. 26p.

YULIANG, Q.; BUZHOU, M.; JIULIANG, F. Study on monitoring farmland by using remote sensing and GIS in Shanxi China. Advances in Space Research, Oxford, v.26, n.7, p.1059-1064, 2000. 